\title{
Sliding Mode Control for Uncertain Chaotic Systems with Nonlinear Inputs
}

\author{
Yongchun Liu ${ }^{1} \&$ Da $\operatorname{Lin}^{1}$ \\ ${ }^{1}$ School of Automatic and Electronic Information, Sichuan University of Science and Engineering, 180 Xue Yuan \\ Street, Zigong 643000, China \\ Correspondence: Da Lin, School of Automatic and Electronic Information, Sichuan University of Science and \\ Engineering, 180 Xue Yuan Street, Zigong 643000, China. Tel: 86-136-2902-2103. E-mail: \\ linda_740609@yahoo.cn
}

Received: October 23, 2011

Accepted: November 17, $2011 \quad$ Online Published: May 24, 2012

doi:10.5539/mer.v2n1p1

URL: http://dx.doi.org/10.5539/mer.v2n1p1

The research is supported by the Youth Foundation of Sichuan Provincial Education Department (No. 11ZB097), the Talents Project of Sichuan University of Science and Engineering (No. 2011RC07)

\begin{abstract}
A sliding mode control design for a class of uncertain chaotic systems subject to sector nonlinear inputs and dead zone is considered in this paper. It is guaranteed that, under the proposed control law, chaotic systems can asymptotically drive the system orbits to arbitrarily desired trajectories even with both uncertainties and input nonlinearities. However, in the sliding mode, the investigated uncertain chaotic system with nonlinear input still possesses advantages of fast response, good transient performance and insensitive to the parameter uncertainties and external disturbances as the systems with linear input. Finally, the Duffing-Holmes chaotic system and the multi-scroll chaotic system are used as an illustrative example to demonstrate the effectiveness of the proposed control scheme.
\end{abstract}

Keywords: uncertain chaotic system, sliding mode control, sector nonlinearity, dead-zone

\section{Introduction}

Over many years, chaos has been shown to be an interesting and even common phenomenon in nature. Chaotic systems are characterized by two defining properties: 1) Sensitive Dependence to Initial Conditions, 2) Transitivity (Devaney, 1989; Martelli et al., 1998). Chaos has been shown to exist in a wide variety of settings: in fluid dynamics such as Raleigh-Bernard convection, in chemistry such as the Belousov-Zhaobitinsky reaction, in nonlinear optics in certain lasers, in celestial mechanics, in electronics such as Chua's circuit, in the flutter of an overdriven airplane wing, some models of population dynamics, and likely in meteorology, physiological oscillations such as certain heart rhythms as well as brain patterns. A complete list of natural systems which can potentially become chaotic would be too large to publish here. Nonetheless, chaos was not considered a desirable property in engineering control practice.

In 1990, Ott, Grebogi, and Yorke (1990) (OGY) published a paper which most importantly, to this discussion, served as a case study which dispelled the paradigm that chaos is undesirable. Chaos control is an important topic in the nonlinear science. In essence, chaos control is guiding a chaotic system to reach a desired goal dynamics via various controllers. Chaos control approaches can be classified into two categories, namely feedback and non-feedback methods (Soong et al., 2007). The feedback methods stabilize one of the unstable periodic orbits embedded in its chaotic attractor by applying a small time-dependent perturbation proportional to the deviation between the desired and the actual trajectories calculated at every time instant. Such as occasional proportional feedback technique (Hunt, 1991), delayed feedback method (Pyragas, 1992), neural control (Lin, 2011), adaptive control (Lin et al., 2010), etc., are all typical feedback methods. For the systems in which the state variables are not accessible on-line or high-speed systems, it is difficult to apply the feedback scheme, and non-feedback methods are more appropriate (Lima, 1990; Braiman \& Goldhirsch, 1991; Fronzoni, 1991; Mettin \& Kurz, 1995; Yang, Qu, \& Hu, 1996; Kraus et al., 1996; Guderian et al., 1999; Murali et al., 1995; Jackson, 1990; Rajasekar, 1995; Rajasekar et al., 1997; Ramesh \& Narayanan, 1999; Antoniou \& Bosco, 2000; Lei et al., 
2004).

For designing a robust control, sliding mode control is frequently adopted due to its inherent advantages of easy realization, fast response, good transient performance and insensitive to variation in plant parameters or external disturbances. The sliding mode control has been successfully applied to cope with the orbit tracking problem for uncertain chaotic systems (Nastaran \& Ali, 2009; Wallace et al., 2009; Hassan \& Aria, 2009). The slide mode controller for uncertain chaotic systems with input nonlinearities are proposed (Yan, 2007; Wang et al., 2009).

On the basis of (Yan, 2007; Wang et al., 2009), the tracking problem for uncertain chaotic systems with sector nonlinearity and dead zones is considered. Sliding mode control is applied to control the system. The advantage of such control is that, when sliding, the system is robust to system uncertainties and external disturbance. Based on the proposed method, a switching manifold is designed for chaos control. In particular, the chaotic systems can be driven to arbitrary trajectory, even when the desired trajectories are not located on the embedded orbits of a chaotic system. Finally, computer simulation for the uncertain Duffing-Holmes system and the multi-scroll chaotic attractors are included to verify and visualize the effect of the sliding mode control.

\section{Notation and Problem Formulation}

Throughout this paper, it is noted that, $\lambda(W)$ denotes an eigenvalue of $W$ and $\lambda_{\max }(W)$ represents the $\max \left[\lambda_{i}(W)\right], i=1, \ldots, n|w|$ epresents the absolute value of $w$ and $\|W\|$ represents the Euclidean norm when $W$ is a vector or the induced norm when $W$ is a matrix. $\operatorname{sign}(s)$ is the sign function of $s$, if $s>0, \operatorname{sign}(s)=$ 1 ; if $s=0, \operatorname{sign}(s)=0$; if $s<0, \operatorname{sign}(s)=-1$.

In this paper, we consider a class of uncertain chaotic systems with sector nonlinearities and dead zones described as

$$
\left\{\begin{array}{l}
\dot{x}_{i}=x_{i+1}, \quad 1 \leq i \leq n-1, \\
\dot{x}_{n}=f(\boldsymbol{X}, t)+\Delta f(\boldsymbol{X}, t)+\phi(u(t))+\delta(t),
\end{array}\right.
$$

Where $X(t)=\left[x_{1}(t), x_{2}(t), \ldots, x_{n}(t)\right]=\left[x(t), \dot{x}(t), \ldots, x^{n-1}(t)\right] \in R^{n}$ is the state vector, $f(x, t) \in R$ is a given nonlinear function of $\boldsymbol{X}$ and $\boldsymbol{t}, u(t) \in R$ is the control input, $\Delta f(x, t)$ is the parameter uncertainty applied to the system, and $\delta(t)$ denotes the external noise perturbation. The superscript $n$ denotes the order of differentiation. System (1) is most often applied in mechanical systems such as the Duffing-Holmes damped spring system, Van der Pol equation, robot systems and flexible-joint mechanisms (Slotine \& Li, 1991). The function $\phi(u(t))$ denotes dead-zone nonlinearity described by

$$
\left\{\begin{array}{l}
\beta_{1}\left(u(t)-u_{0+}\right)^{2} \leq\left(u(t)-u_{0+}\right) \phi(u(t)) \leq \beta_{2}\left(u(t)-u_{0+}\right)^{2}, u(t)>u_{0+} \\
\phi(u(t))=0,-u_{0-} \leq u(t) \leq u_{0+} \\
\beta_{1}\left(u(t)+u_{0-}\right)^{2} \leq\left(u(t)+u_{0-}\right) \phi(u(t)) \leq \beta_{2}\left(u(t)+u_{0-}\right)^{2}, u(t)<-u_{0-}
\end{array}\right.
$$

where $\beta_{1}, \beta_{2}>0$. A nonlinear function $\phi(u(t))$ is illustrated in Figure 1. Generally, the parameter uncertainty $\Delta f(x, t)$ and the noise perturbation $\delta(t)$ are assumed bounded, i.e.

$$
|\Delta f(\boldsymbol{X}, t)| \leq \alpha_{1}\|\boldsymbol{X}\|, \quad|\delta(t)|<\alpha_{2},
$$

where $\alpha_{1}, \alpha_{2}>0$ are given.

The control task is to force the system (1) to track an n-dimension desired vector $X_{d}(t)$, $X_{d}(t)=\left[x_{d 1}(t), x_{d 2}(t), \ldots, x_{d n}(t)\right]=\left[x_{d}(t), \dot{x_{d}}(t), \ldots, x_{d}{ }^{n-1}(t)\right]$, which belong to a class of $\boldsymbol{C}$ functions on $\left[\mathrm{t}_{0}\right.$, $\left.{ }_{\infty}\right]$. Let us define the tracking error as

$$
\begin{aligned}
\boldsymbol{E}(t)=\boldsymbol{X}(t)-\boldsymbol{X}_{d}(t) & =\left[x(t)-x_{d}(t), \dot{x}(t)-\dot{x}_{d}(t), \ldots, x^{(n-1)}(t)-x_{d}^{(n-1)}(t)\right] \\
& =\left[e(t), \dot{e}(t), \ldots, e^{(n-1)}(t)\right] \\
& =\left[e_{1}(t), e_{2}(t), \ldots, e_{n}(t)\right]
\end{aligned}
$$

The control goal considered in this paper is that for any given orbit $X_{d}(t)$, a sliding mode control is designed in spite of the nonlinearity in control input, such that the resulting tracking error satisfies 


$$
\lim _{t \rightarrow \infty}\|\boldsymbol{E}(t)\|=\lim _{t \rightarrow \infty}\left\|\boldsymbol{X}(t)-\boldsymbol{X}_{d}(t)\right\|=0 .
$$

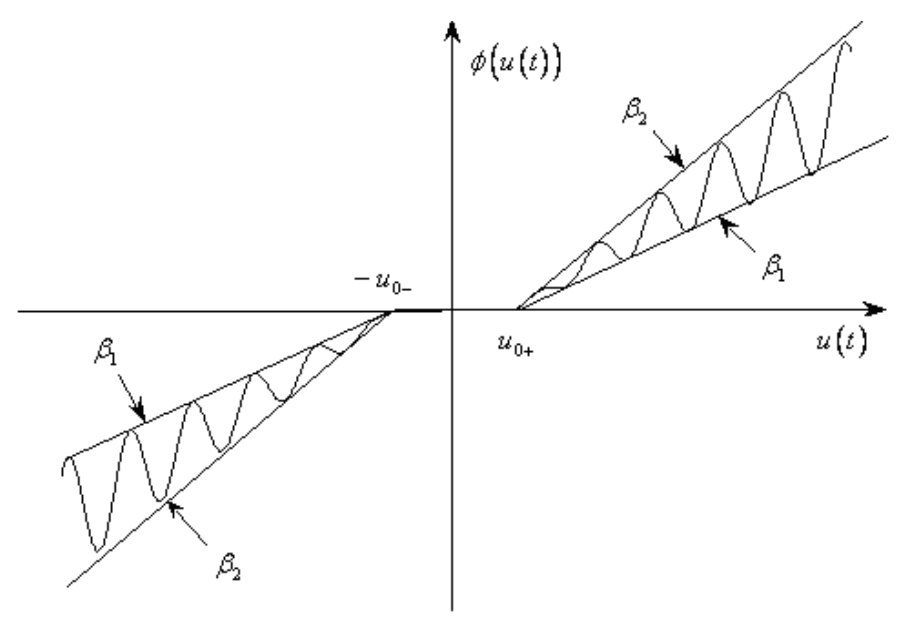

Figure 1. The nonlinear function $\phi(u(t))$

In order to achieve the above control goal for uncertain chaotic systems with nonlinear input, according to variable structure control theory, there exist two basic steps. First, it need to select an appropriate switching surface for the system such that the sliding motion on the manifold can result in $\lim _{t \rightarrow \infty}\|\boldsymbol{E}(t)\|=0$. Second, it needs to determine a control law such that the existence of the sliding mode can be guaranteed, even under the influence of input nonlinearity.

Now, the sliding surface is define as

$$
s(t)=e_{n}(t)+\sum_{i=1}^{n-1} c_{i} e_{i}(t),
$$

where $s(t) \in R$ and $c_{i}(i=1,2, \ldots, n-1)$ are design parameters which can be easily determined later. When the system operates in the sliding mode, it satisfies the following equations (Utkin, 1978; Itkis, 1976)

$$
\begin{gathered}
s(t)=e_{n}(t)+\sum_{i=1}^{n-1} c_{i} e_{i}(t)=0, \\
\dot{s}(t)=\dot{e}_{n}(t)+\sum_{i=1}^{n-1} c_{i} \dot{e}_{i}(t)=0 .
\end{gathered}
$$

From Eqs. (4) and (7) the following sliding mode dynamics can be obtained as

$$
\begin{gathered}
\left\{\begin{array}{l}
\dot{e}_{i}(t)=e_{i+1}(t)(1 \leq i \leq n-2) \\
\dot{e}_{n-1}(t)=e_{n}(t)=-\sum_{i=1}^{n-1} c_{i} e_{i}(t)
\end{array}\right. \\
\dot{e}_{n}(t)=\dot{x}_{n}(t)-\dot{x}_{d n}(t)=f(X, t)+\Delta f(X, t)+\phi(u(t))+\delta(t)-x_{d}^{n}(t) .
\end{gathered}
$$

Eq. (8) can be also in a matrix equation form as 


$$
\left[\begin{array}{c}
\dot{e}_{1} \\
\dot{e}_{2} \\
\vdots \\
\dot{e}_{n-1}
\end{array}\right]=\left[\begin{array}{cccccc}
0 & 1 & 0 & \cdots & \cdots & 0 \\
0 & 0 & 1 & 0 & \cdots & 0 \\
\vdots & \vdots & \vdots & \ddots & \ddots & \vdots \\
-c_{1} & -c_{2} & \cdots & \cdots & \cdots & -c_{n-1}
\end{array}\right]\left[\begin{array}{c}
e_{1} \\
e_{2} \\
\vdots \\
e_{n-1}
\end{array}\right]=\boldsymbol{M}\left[\begin{array}{c}
e_{1} \\
e_{2} \\
\vdots \\
e_{n-1}
\end{array}\right]
$$

Obviously, the design parameters $c_{i}(i=1,2, \ldots, n-1)$ can be determined such that $\lambda_{\max }(M)<0$.. Once $c_{i}(i=1,2, \ldots, n-1)$ is determined to result in $\lambda_{\max }(M)<0$, the stability of Eq. (10) is surely guaranteed. Furthermore, by Eq. (7a), $e_{n}(t)$ is also stable. Meanwhile, it is worthy of note that these eigenvalues of matrix $\boldsymbol{M}$ are also relative to the speed of system response. By surveying Eq. (10), it reveals that when the controlled system is in the sliding mode, the system is insensitive to parameter uncertainties, external disturbances and input nonlinearity. In other word, the controlled chaotic system is robust.

\section{Design Sliding Mode Control}

Before stating the scheme of the controller, the reaching condition of the sliding mode is given below.

Lemma 1. The motion of the sliding mode (6) is asymptotically stable, if the following reaching condition is held

$$
s(t) \dot{s}(t)<0 .
$$

Proof. Let $V(t)=0.5 s^{2}(t)$ be the Lyapunov function. According to Lyapunov stability theory, condition (11) ensures that

$$
\dot{V}(t)=s(t) \dot{s}(t)<0 .
$$

Then, $s(t)$ is toward the switching surface and the sliding mode (6) is asymptotically stable. $\square$ To achieve the reaching condition indicated in Lemma 1, a control law is proposed as

$$
u(t)=\left\{\begin{array}{l}
-r \eta \operatorname{sign}(s(t))-u_{0-}, s(t)>0 \\
0, s(t)=0 \quad, \quad r>1 / \beta_{1}, \\
-r \eta \operatorname{sign}(s(t))+u_{0+}, s(t)<0
\end{array},\right.
$$

where $\eta=\sum_{i=1}^{n-1}\left|c_{i} e_{i+1}(t)\right|+|f(\boldsymbol{X}, t)|+\alpha_{1}\|\boldsymbol{X}\|+\alpha_{2}+\left|x_{d}^{(n)}(t)\right|$.

In the following, the proposed scheme (13) will be proved to be able to derive the uncertain chaotic system (1) with nonlinear input onto the sliding mode $s(t)=0$.

Theorem 1. Consider uncertain chaotic systems (1) with nonlinear input. The hitting condition (11) of the sliding mode is satisfied, if the control $u(t)$ is given by (13).

Proof. Substituting Eqs. (8) and (9) into the derivative $s(t) \dot{s}(t)$, we get the following result.

$$
\begin{aligned}
s(t) \dot{s}(t) & =s(t)\left[\left(\sum_{i=1}^{n-1} c_{i} \dot{e}_{i}(t)\right)+\dot{e}_{n}(t)\right] \\
& =s(t)\left[\sum_{i=1}^{n-1} c_{i} e_{i+1}(t)+f(\boldsymbol{X}, t)+\Delta f(\boldsymbol{X}, t)+\delta(t)+\phi(u(t))-x_{d}^{(n)}(t)\right], \\
& \leq \mid s(t)\left[\left[\sum_{i=1}^{n-1}\left|c_{i} e_{i+1}(t)\right|+|f(X, t)|+\alpha_{1}|| \boldsymbol{X} \|+\alpha_{2}+\left|x_{d}^{(n)}(t)\right|\right]+s(t) \phi(u(t))\right.
\end{aligned}
$$

From Eqs. (2) and (13), we have

if $s(t)>0$, then

$$
\left(u(t)+u_{0-}\right) \phi(u(t))=-r \eta \operatorname{sign}(s(t)) \phi(u(t)) \geq \beta_{1}\left(u(t)+u_{0-}\right)^{2}=\beta_{1} r^{2} \eta^{2}[\operatorname{sign}(s(t))]^{2} .
$$


if $s(t)<0$, then

$$
\left(u(t)-u_{0+}\right) \phi(u(t))=-r \eta \operatorname{sign}(s(t)) \phi(u(t)) \geq \beta_{1}\left(u(t)-u_{0+}\right)^{2}=\beta_{1} r^{2} \eta^{2}[\operatorname{sign}(s(t))]^{2} .
$$

From Eqs. (15) and (16), we have

$$
-r \eta \operatorname{sign}(s(t)) \phi(u(t)) \geq \beta_{1} r^{2} \eta^{2}[\operatorname{sign}(s(t))]^{2} .
$$

Multiplying $s^{2}(t)$ both sides of Eq. (17), we have

$$
\begin{aligned}
& -r \eta s^{2}(t) \operatorname{sign}(s(t)) \phi(u(t)) \geq \beta_{1} r^{2} \eta^{2} s^{2}(t)[\operatorname{sign}(s(t))]^{2} \\
& \quad \Rightarrow-r \eta s(t) \phi(u(t)) \geq \beta_{1} r^{2} \eta^{2}|s(t)| \\
& \quad \Rightarrow s(t) \phi(u(t)) \leq-\beta_{1} r \eta|s(t)|
\end{aligned}
$$

By placing Eq. (18) into Eq. (14), we get

$$
s(t) \dot{s}(t) \leq\left(1-\beta_{1} r\right) \eta|s(t)|
$$

Since $r>\beta_{1}$ has been selected in Eq. (13), one can conclude that the reaching condition $s(t) \dot{s}(t)<0$ is always satisfied. Thus the proof is achieved completely.

\section{Computer Simulations}

\subsection{Controlling the Uncertain Duffing-Holmes System}

In this subsection, an uncertain Duffing-Holmes system is considered. The Duffing-Holmes system can be described by

$$
\left\{\begin{array}{l}
\dot{x}_{1}=x_{2} \\
\dot{x}_{2}=-p_{1} x_{1}-p x_{2}-x_{1}^{3}+q \cos (\omega t)
\end{array}\right.
$$

which can present a nonlinear damped vibration under a periodic force excitation. The chaotic motion of the Duffing-Holmes system is shown in Figure 2, with parameters $p=0.25, p_{1}=-1.0, q=0.3$ and $\omega=1.0$.

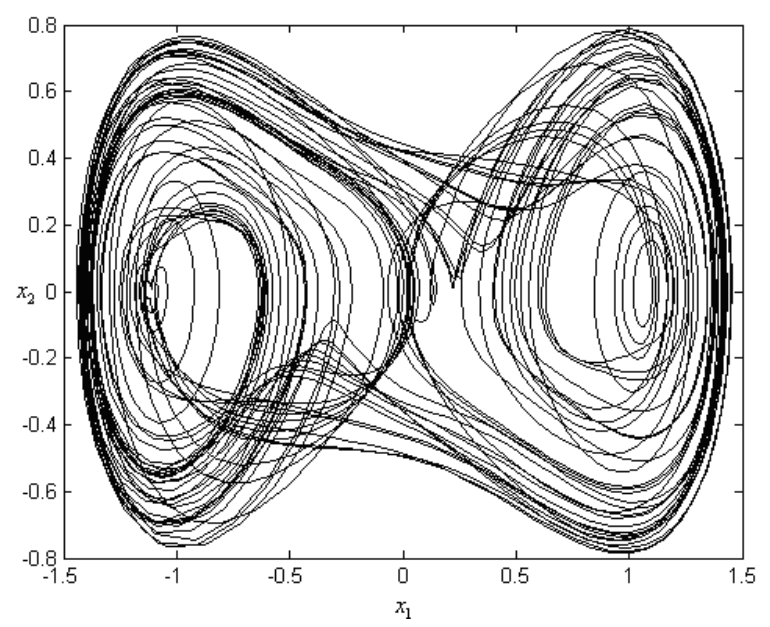

Figure 2. The chaotic attractor of Duffing-Holmes system

Consider the system (20) is perturbed by an uncertainty term and external noise, added to its second equation. The uncertain Duffing-Holmes system with nonlinear input, which can be written as 


$$
\left\{\begin{array}{l}
\dot{x}_{1}=x_{2} \\
\dot{x}_{2}=-p_{1} x_{1}-p x_{2}-x_{1}^{3}+q \cos (\omega t)+\Delta f(\boldsymbol{X}, t)+\delta(t)+\phi(u(t))
\end{array}\right.
$$

In order to demonstrate the robustness of the sliding mode controller, suppose $\Delta f(x, t)$ and $\delta(t)$ satisfy, respectively, $|\Delta f(X, t)| \leq \alpha_{1}\|\mathrm{X}\|=0.1\|\mathrm{X}\|$ and $|\delta(t)| \leq \alpha_{2}=0.2$. The nonlinear input is defined as

$$
\phi(u(t))=\left\{\begin{array}{l}
(0.7+0.2 \sin (u(t)))\left(u(t)-u_{0+}\right), u(t)>u_{0+} \\
0,-u_{0-} \leq u(t) \leq u_{0+} \\
(0.7+0.2 \sin (u(t)))\left(u(t)+u_{0-}\right), u(t)<-u_{0-}
\end{array},\right.
$$

where $u_{0+}=u_{0-}=6$ denote the dead zone, based on Eq. (22) $\beta_{1}=0.5, \beta_{2}=0.9$ can be obtained. We choose $r=3$ to satisfy $r>1 / \beta_{1}=2$. The control goal is to drive the uncertain chaotic system (21) to the following trajectory

$$
x_{d}(t)=A \sin (\theta t),
$$

where $A=1.2, \theta=0.8$. According to Eq. (10), we select $c_{1}=3$ to result in a stable sliding mode. According to Eqs. (6) and (13), we design the controller $u(t)$. To explicitly show the control effect of the proposed method, the time interval is divided into two parts: At the first stage of the simulation, no control input is applied; the control input is then activated thereafter.

Figures 3 and 4 show the simulation results with initial value $\boldsymbol{X}_{0}=\left[\begin{array}{ll}1 & 1\end{array}\right]$. The control input is activated at 5 sec, from Figures 3 and 4, the controlled system (21) is driven to the desired trajectories, and is insensitive to uncertainty of chaotic system, external disturbances, dead zones and sector nonlinear input. In other words, the controlled chaotic system (21) is robust.
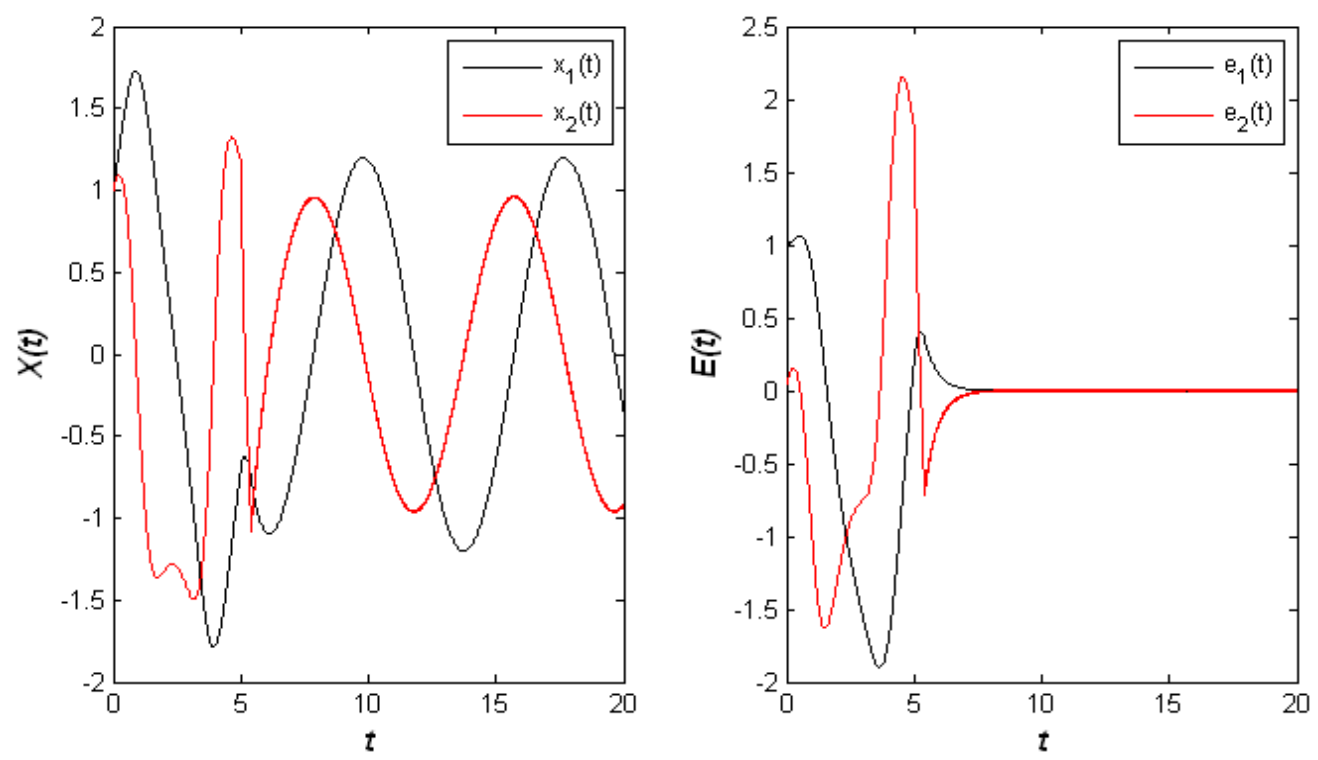

Figure 3. The state responses of system (20) and its error responses 


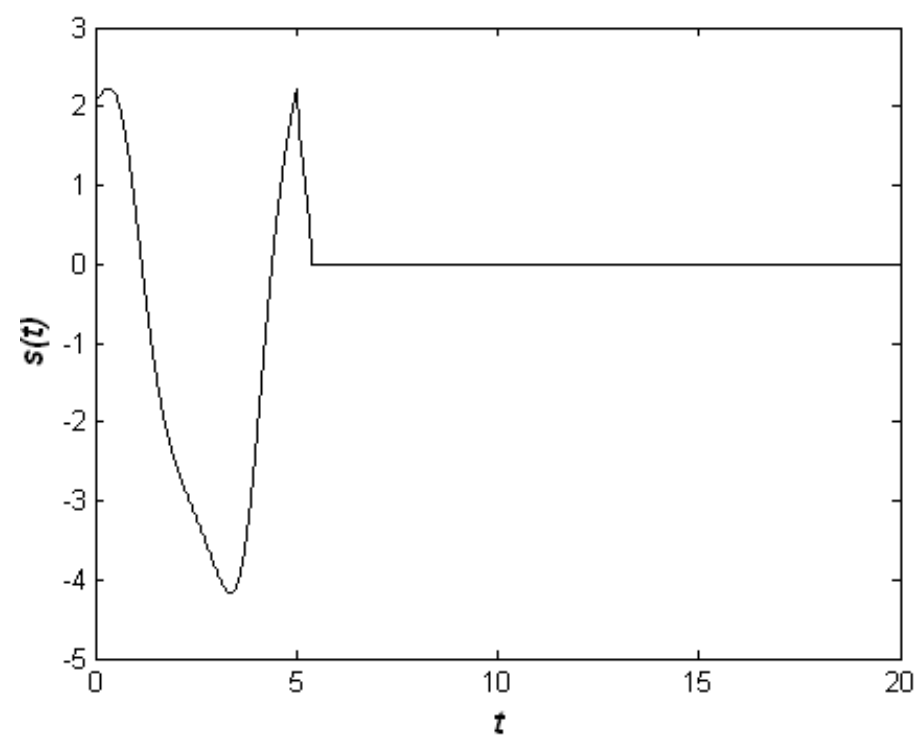

Figure 4. Time response of $s(t)$

\subsection{Controlling the Uncertain Multi-scroll Chaotic Attractors}

Since Suykens and Vandewalle (Suykens \& Vandewalle, 1991, 1993a, 1993b) first introduced a family of n-double scroll chaotic attractor from the so-called quasi-linear function approach, many different approaches or techniques have been proposed to generate multi-scroll chaotic attractors, such as saturated function series method, hysteresis series switching method, thresholding approach, among many others (Lü, Chen, \& Yu, 2004a, 2004b; Lü et al., 1998; Lü \& Chen, 2006). More recently, Lü et al. (Lü, Chen, \& Yu, 1998; Yu, Lü, \& Chen, ) implemented the multi-scroll chaotic attractors and the multi-torus chaotic attractors by analog circuits successfully. Multi-scroll chaotic attractors have many practical applications in, for example, broadband signal generation, secure and digital communications, and perhaps efficient liquid mixing, to name a few from among other. So, it is necessary and important to study multi-scroll chaotic attractors' control.

In this subsection, an uncertain multi-scroll chaotic attractors from saturated function series is considered. The multi-scroll chaotic attractors (Lü, Chen, \& Yu, 2004a) can be described by

$$
\left\{\begin{array}{l}
\dot{x}_{1}=x_{2} \\
\dot{x}_{2}=x_{3} \\
\dot{x}_{3}=-a x_{1}-b x_{2}-c x_{3}+d_{1} f\left(x_{1} ; k, h, p, q\right)
\end{array},\right.
$$

where $a, b, c$, and $d_{1}$ are positive constants. Saturated function $f\left(x_{1} ; k, h, p, q\right)$ is defined as follows

$$
f\left(x_{1} ; k, h, p, q\right)=\left\{\begin{array}{l}
(2 q+1) k, \quad \text { if } x_{1}>q h+1 \\
k\left(x_{1}-i h\right)+2 i k, \quad \text { if }\left|x_{1}-i h\right| \leq 1,-p \leq i \leq q \\
(2 i+1) k, \quad \text { if } i h+1<x_{1}<(i+1) h-1,-p \leq i \leq q-1 \\
-(2 p+1) k, \quad \text { if } x_{1}<-p h-1
\end{array} .\right.
$$

Figure 5 shows a 6-scroll chaotic attractors of system (24), where $a=b=c=d_{1}=0.7, k=9, h=18$, $p=2, q=2$. 


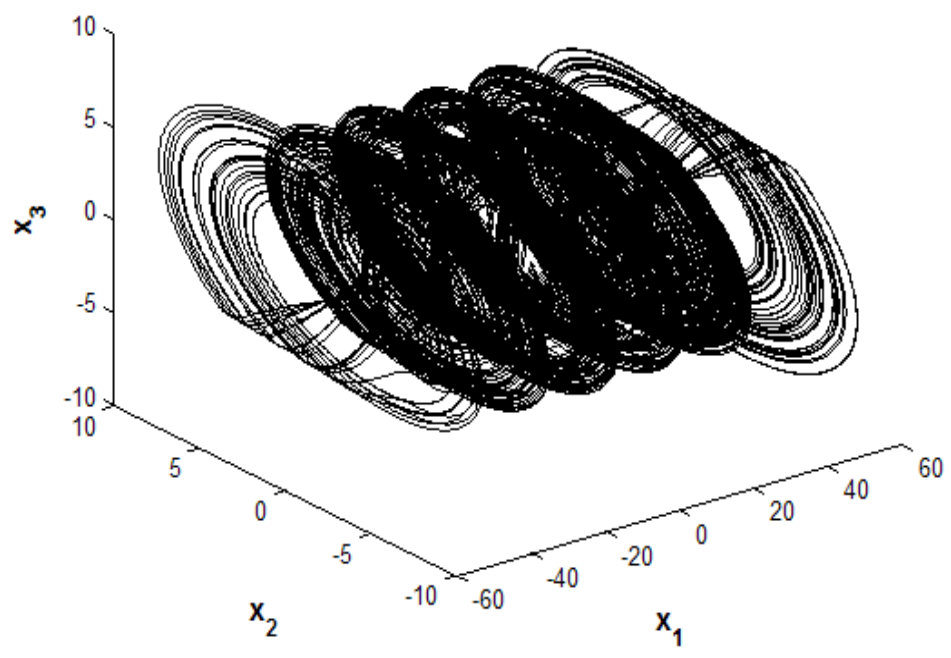

Figure 5. 6-scroll chaotic attractors

Consider the system (24) is perturbed by an uncertainty term and external noise, added to its third equation. The uncertain multi-scroll chaotic attractors with nonlinear input, which can be written as

$$
\left\{\begin{array}{l}
\dot{x}_{1}=x_{2} \\
\dot{x}_{2}=x_{3} \\
\dot{x}_{3}=-a x_{1}-b x_{2}-c x_{3}+d_{1} f\left(x_{1} ; k, h, p, q\right)+\Delta f(X, t)+\delta(t)+\phi(u(t))
\end{array}\right.
$$

In order to demonstrate the robustness of the sliding mode controller, suppose $\Delta f(x, t)$ and $\delta(t)$ satisfy, respectively, $|\Delta f(X, t)| \leq \alpha_{1}\|\mathrm{X}\|=0.2\|\mathrm{X}\|$ and $|\delta(t)| \leq \alpha_{2}=0.3$. The nonlinear input is defined by Eq. (22), and the goal trajectory is given by Eq. (23), respectively, $A=10$ and $\theta=1.2$. According to (10), we select $c_{1}=2, c_{2}=2$ to result in a stable sliding mode. According to Eqs. (6) and (13), we design the controller $u(t)$. To explicitly show the control effect of the proposed method, the time interval is divided into two parts: At the first stage of the simulation, no control input is applied; the control input is then activated thereafter.

The simulation results with initial value $\boldsymbol{X}_{0}=\left[\begin{array}{lll}6 & -2 & 8\end{array}\right]$ are shown in Figures 6 and 7. The control input is activated at $5 \mathrm{sec}$, Figure 6 shows the state responses of system (26) and its error, and Figure 7 shows the corresponding $s(t)$. From Figures 6 and 7, the controlled system (26) is driven to the desired trajectories, and is insensitive to uncertainty of chaotic system, external disturbances, dead zones and sector nonlinear input.
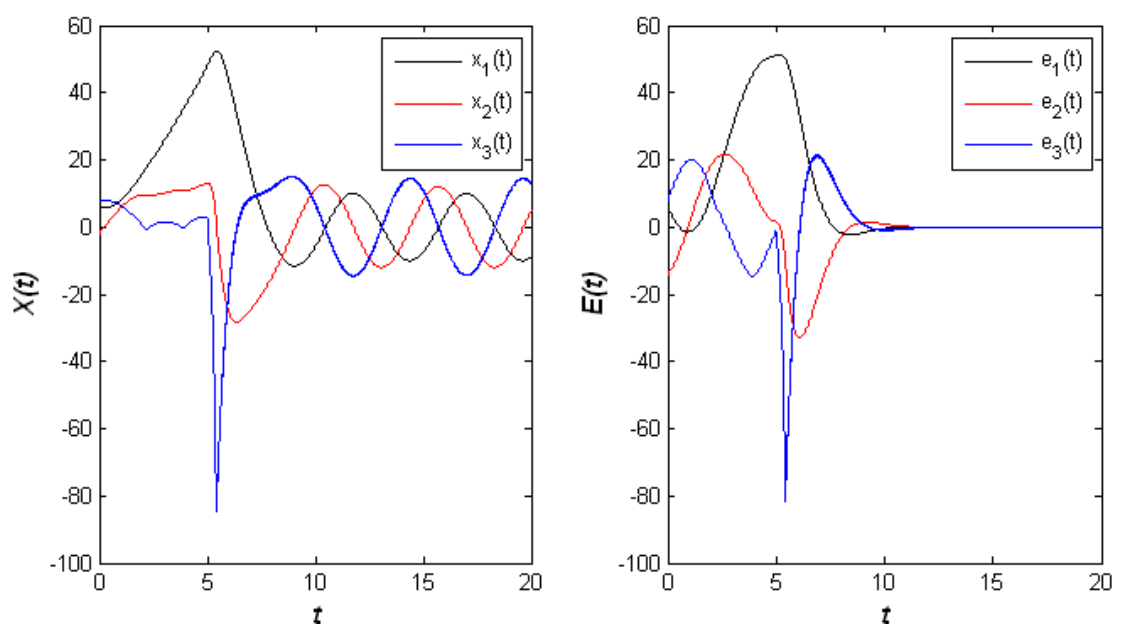

Figure 6. The state responses of system (25) and its error responses 


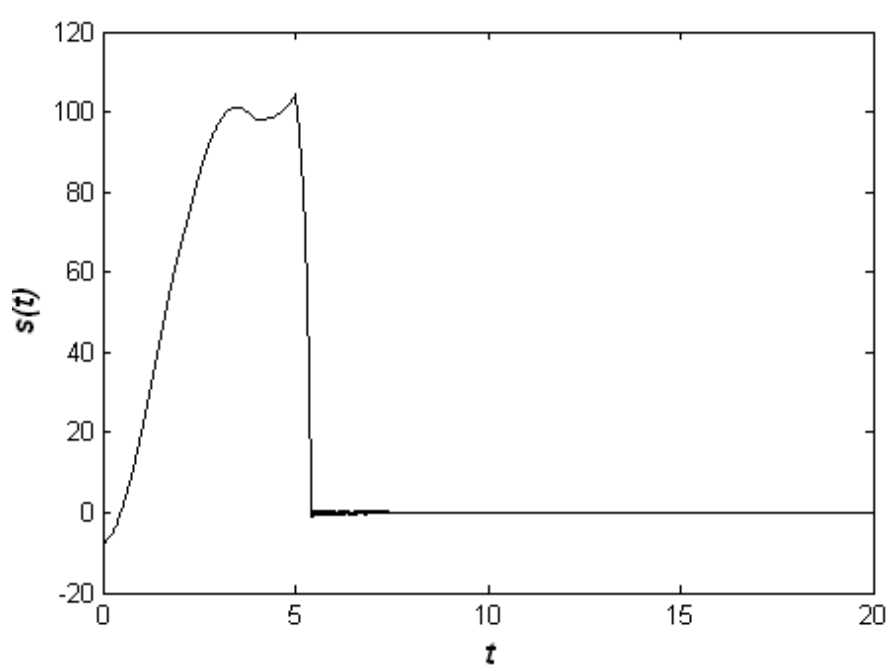

Figure 7. Time response of $s(t)$

\section{Conclusions}

In this paper, a robust control design for a class of uncertain chaotic systems with sector nonlinearities has been proposed. A newly developed sliding mode controller has been proposed and applied on a well-known Duffing-Holmes system and the multi-scroll chaotic attractors. This proposed sliding mode control is able to overcome the parameter uncertainty, external noise, sector nonlinearity and dead zone. Both analysis and simulation reveal that the proposed sliding mode control has great potential for controlling uncertain chaotic dynamics, even for higher-dimensional and more complex systems.

\section{References}

Antoniou, I., \& Bosco, F. (2000). Probabilistic control of chaos through small perturbation. Chaos, Solitons \& Fractals, 11(1-3). 359-371. http://dx.doi.org/10.1016/S0960-0779(98)00306-3

Braiman, Y., \& Goldhirsch, I. (1991). Taming chaotic dynamics with weak periodic perturbations. Physical Review Letters, 66 (20), 2545-2548. http://dx.doi.org/10.1103/PhysRevLett.66.2545

Devaney, R. L. (1989). An Introduction to Chaotic Dynamical Systems (2nd ed.). CA: Addison Wesley, Redwood City.

Fronzoni, L., Giocondo, M., \& Pettini, M. (1991). Experimental evidence of suppression of chaos by resonant $\begin{array}{lllll}\text { parametric } & \text { perturbations. } & \text { Physical }\end{array}$ http://dx.doi.org/10.1103/PhysRevA.43.6483

Guderian, A., Münster, A. F., Jinguji, M., Kraus, M., \& Schneider, F. W. (1999). Resonant chaos control by light in a chemiluminescent reaction. Chemical Physics Letters, 312(5, 6), 440-446.

Hassan, S., \& Aria, A. (2009). Control of stochastic chaos using sliding mode method. Journal of Computational and Applied Mathematics, 225(1), 135-145.

Hunt, E. R. (1991). Stabilizing high-period orbits in a chaos system: the diode resonator. Physical Review Letters, 67(15), 1953-1955. http://dx.doi.org/10.1103/PhysRevLett.67.1953

Itkis, U. (1976). Control System of Variable Structure. New York: Wiley.

Jackson, E. A. (1990). The entrainment and migration controls of multiple-attractor systems. Physics Letters A, 151(9). 478-484. http://dx.doi.org/10.1016/0375-9601(90)90465-Z

Kraus, M., Müller, J., Lebender, D., \& Schneider, F. W. (1996). Resonant chaos control by periodic perturbations. Chemical Physics Letters, 260(1,2). 51-58.

Lei, Y., Xu, W., Xu, Y., \& Fang, T. (2004). Chaos control by harmonic excitation with proper random phase. Chaos, Solitons \& Fractals, 21(5). 1175-1181. http://dx.doi.org/10.1016/j.chaos.2003.12.086

Lima, R., \& Pettini, M. (1990). Suppression of chaos by resonant parametric perturbations. Physical Review A, 41(2). 726-733. http://dx.doi.org/10.1103/PhysRevA.41.726

Lin, D. (2011). Self-organizing adaptive fuzzy neural control for the synchronization of uncertain chaotic 
systems with random-varying parameters. Neurocomputing, 74(12-13), 2241-2249. http://dx.doi.org/10.1016/j.neucom.2011.03.003

Lin, D., Wang, X., Nian, F., \& Zhang, Y. (2010). Dynamic fuzzy neural networks modeling and adaptive backstepping tracking control of uncertain chaotic systems. Neurocomputing, 73(16-18), 2873-2881. http://dx.doi.org/10.1016/j.neucom.2010.08.008

Lü, J., \& Chen, G. (2006). Generating multi-scroll chaotic attractors: theories, methods and applications. International Journal of Bifurcation and Chaos, 16(4), $775-858$. http://dx.doi.org/10.1142/S0218127406015179

Lü, J., Chen, G., \& Yu, X. (2004a). Leung H. Design and Analysis of Multiscroll Chaotic Attractors From Saturated Function Series. IEEE Transactions on Circuits and Systems I, 51(12), 2476-2490. http://dx.doi.org/10.1109/TCSI.2004.838151

Lü, J., Han, F., Yu, X., \& Chen, G. (2004b). Generating 3-D multi-scroll chaotic attractors: A hysteresis series switching method. Automatica, 40(10), 1677-1687. http://dx.doi.org/10.1016/j.automatica.2004.06.001

Lü, J., Murali, K., Sinha, S., Leung, H., \& Aziz-Alaoui, M. A. (2008). Generating multi-scroll chaotic attractors by thresholding. Physics Letters A, 372(18), 3234-3239. http://dx.doi.org/10.1016/j.physleta.2008.01.065

Lü, J., Yu, S., Leung, H., \& Chen, G. (1998). Experimental verification of multidirectional multiscroll chaotic attractors. IEEE Transactions on Circuits and systems I, 53(1), 149-165.

Martelli, M., Dong, M., \& Seph, T. (1998). Defining chaos. Mathematics Magazine. 71(2), $112-122$. http://dx.doi.org/10.2307/2691012

Mettin, R., \& Kurz, T. (1995). Optimized periodic control of chaotic systems. Physics Letters A, 206(5, 6). 331-339.

Murali, K., Lakshmanan, M., \& Chua, L. O. (1995). Controlling and synchronization of chaos in the simplest dissipative non-autonomous circuit. International Journal of Bifurcation and Chaos. 5(2), 563-571. http://dx.doi.org/10.1142/S0218127495000466

Nastaran, V., \& Ali, K. S. (2009). Chaos control in delayed chaotic systems via sliding mode based delayed feedback. Chaos, Solitons \& Fractals, 40(1). 159-165.

Ott, E. Grebogi, C., \& Yorke, J. A. (1990). Controlling chaos. Physical Review Letters, 64(11), $1196-1199$. http://dx.doi.org/10.1103/PhysRevLett.64.1196

Pyragas, K. (1992). Continuous control of chaos by self-controlling feed back. Physics Letters A, 170(6), 421-428. http://dx.doi.org/10.1016/0375-9601(92)90745-8

Rajasekar, S. (1995). Controlling of chaotic motion by chaos and noise signals in a logistic map and a Bonhoeffer-Van der Pol oscillator. Physical Review E, 51(1), 775-778. http://dx.doi.org/10.1103/PhysRevE.51.775

Rajasekar, S., Murali, K., \& Lakshmanan, M. (1997). Control of chaos by nonfeedback methods in a simple electronic circuit system and the FitzHugh-Nagumo equation. Chaos, Solitons \& Fractals, 8(9), 1545-1558. http://dx.doi.org/10.1016/S0960-0779(96)00154-3

Ramesh, M., \& Narayanan, S. (1999). Chaos control by nonfeedback methods in the presence of noise. Chaos, Solitons \& Fractals, 10(9), 1473-1489. http://dx.doi.org/10.1016/S0960-0779(98)00132-5

Slotine, J. E., \& Li, W. (1991). Applied Nonlinear Control. Englewood Cliffs: Prentice-Hall.

Soong, C. Y., Huang, W. T., \& Lin, F. P. (2007). Chaos control on autonomous and non-autonomous systems with various types of genetic algorithm-optimized weak perturbations. Chaos, Solitons \& Fractals, 34(5), 1519-1537. http://dx.doi.org/10.1016/j.chaos.2006.04.023

Suykens, J. A. K., \& Vandewalle, J. (1991). Quasilinear approach to nonlinear systems and the design of n-double scroll $(\mathrm{n}=1,2,3,4, \ldots)$. IEEE Proc. $G$ 138, 595-603.

Suykens, J. A. K., \& Vandewalle, J. (1993a). Between n-double sinls and n-double scrolls (n = 1,2,3,4,..). Proc. Int. Symp. Nonlinear Theory and Its Applications (NOLTA'93), December 5-10, Hawaii, USA, 829-834.

Suykens, J. A. K., \& Vandewalle, J. (1993b). Generation of $\mathrm{n}$-double scrolls $(\mathrm{n}=1,2,3,4, \ldots)$. IEEE Transactions on Circuits and Systems I, 40(11), 861-867. http://dx.doi.org/10.1109/81.251829

Utkin, V. I. (1978). Sliding Mode and their Applications in Variable Structure System. Moscow: Mir Editors. 
Wallace, M. B., Aline, S. P., \& Marcelo, A. S. (2009). Chaos control using an adaptive fuzzy sliding mode controller with application to a nonlinear pendulum. Chaos, Solitons \& Fractals, 42(2), 784-791.

Wang, X. Y., Lin, D., \& Wang, Z. J. (2009). Controlling the uncertain multi-scroll critical chaotic system with input nonlinear using sliding mode control. Modern Physics Letters B, 23(16). 2021-2034. http://dx.doi.org/10.1142/S0217984909020187

Yan, J. J. (2004). Design of robust controllers for uncertain chaotic systems with nonlinear inputs. Chaos, Solitons \& Fractals, 19(3), 541-547. http://dx.doi.org/10.1016/S0960-0779(03)00123-1

Yang, J., Qu, Z., \& Hu, G. (1996). Duffing equation with two periodic forcings: the phase effect. Physical Review E, 53(5), 4402-4413. http://dx.doi.org/10.1103/PhysRevE.53.4402

Yu, S. Lü, J., \& Chen, G. (2007). Theoretical design and circuit implementation of multidirectional multi-torus chaotic attractors. IEEE Transactions on Circuits and systems I, 54(9), 2087-2098. 